Cipango $\begin{aligned} & \text { Cipango } \\ & \text { Cahiers d'études japonaises }\end{aligned}$

Hors-série | 2008

Autour du Genji monogatari

\title{
Du Kagerō no nikki au Genji monogatari
}

From Kagerō no nikki to Genji monogatari

\section{Jacqueline Pigeot}

\section{(2) OpenEdition}

Journals

Édition électronique

URL : https://journals.openedition.org/cipango/592

DOI : 10.4000/cipango.592

ISSN : 2260-7706

Éditeur

INALCO

Édition imprimée

Date de publication : 1 janvier 2008

Pagination : 69-87

ISBN : 978-2-85831-170-5

ISSN : $1164-5857$

Référence électronique

Jacqueline Pigeot, « Du Kagerō no nikki au Genji monogatari », Cipango [En ligne], Hors-série | 2008, mis en ligne le 11 juin 2012, consulté le 30 juin 2021. URL : http://journals.openedition.org/cipango/592 ; DOI : https://doi.org/10.4000/cipango.592

Ce document a été généré automatiquement le 30 juin 2021.

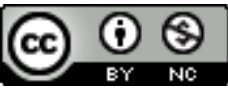

Cipango est mis à disposition selon les termes de la Licence Creative Commons Attribution - Pas d'Utilisation Commerciale 4.0 International. 


\title{
Du Kagerō no nikki au Genji monogatari
}

\author{
From Kagerō no nikki to Genji monogatari
}

Jacqueline Pigeot

1 La communauté scientifique s'accorde aujourd'hui pour penser que le Genji monogatari doit quelque chose au Kagerō no nikki 蛙蛉日記 (Les Mémoires d’une Éphémère) - la première œuvre en prose japonaise qui, du moins pour ce qui nous a été conservé, ait pour auteur une femme.

2 L'auteur des Mémoires, que l'on ne connaît que sous le nom de la «mère de Fujiwara no Michitsuna”藤原道綱母 et Murasaki Shikibu sont séparées par une ou deux générations, la première étant sans doute née en 936, la seconde vers 970 ; elles appartenaient à la même couche sociale, la moyenne noblesse qui fournissait les gouverneurs de province (zuryō) : leurs pères exercèrent tous deux ces fonctions ; tous deux étaient des hommes cultivés, issus de lignées où l'on pratiquait la poésie, et leurs filles bénéficièrent sans aucun doute de leur enseignement et de leur bibliothèque.

Elles furent en contact avec la plus haute aristocratie, à des degrés divers : Murasaki Shikibu servit pendant plusieurs années l'impératrice Shōshi 彰子; la mère de Michitsuna, si elle ne mit guère les pieds à la Cour (peut-être seulement à l'occasion de tel concours de poèmes), fut l'une des épouses d'un membre de la prestigieuse famille qui exerçait alors le pouvoir, Fujiwara no Kaneie 藤原兼家 (929-990). Lors de leur liaison conjugale, Kaneie n'avait pas achevé son ascension politique, mais les Mémoires d'une Éphémère montrent que la mère de Michitsuna entretenait des relations avec ses frères, plus en vue que lui, avec ses sœurs, qui entrèrent au gynécée impérial, et qu'elle connut donc, peu ou prou, les grands de ce monde.

4 Le Genji monogatari et les Mémoires d'une Éphémère peignent donc grosso modo le même monde, reflètent le même code social, le même système de pensée ; ajoutons qu'ils sont écrits dans la même langue, celle qui, pense-t-on, était la langue ordinaire des femmes de la noblesse de la capitale (ces deux derniers mots étant d'ailleurs redondants). 
Cela posé, les deux œuvres reflètent aussi la personnalité, très différente, de leurs auteurs, leur vision du monde particulière, ou la vision du monde qu'elles entendent proposer au lecteur, selon leur dessein propre. On peut dire, pour reprendre un terme un peu facile et galvaudé, que le «monde» (sekai) de leurs œuvres est différent sur quantité de points. Je ne m'étendrai guère sur cette question, mais, peut-être moimême influencée par ces gender studies qui font fureur aux États-unis comme au Japon et dans le crible desquelles sont régulièrement passés les Mémoires d'une Éphémère, je voudrais m'arrêter un instant sur ce qui, à mes yeux, les distingue, s'agissant de ce qu'elles disent, ou montrent, de la femme - ou des femmes.

6 Akiyama Ken a relevé dans le roman quinze propositions générales concernant les femmes, leur sort, leur destin, etc., cela, en général, pour les plaindre ${ }^{1}$. Visiblement, Murasaki Shikibu a mené une certaine réflexion sur la condition féminine. Dans les Mémoires d'une Éphémère, au contraire, on ne peut trouver à ce sujet aucune remarque d'ordre général. L'auteur ne s'intéresse qu'aux individus, elle-même au premier chef. Elle a davantage réfléchi à son propre destin qu'à celui de la femme en soi, et, si elle incrimine beaucoup Kaneie en tant qu'individu, elle ne le fait jamais en tant que représentant du sexe masculin. Elle insiste plutôt sur le fait qu'il n'est pas comme les autres.

7 On remarque par ailleurs qu'aucune des figures de femme du Genji monogatari ne possède la forte personnalité, le goût de l'indépendance de la mère de Michitsuna, telle qu'elle se donne à voir dans les Mémoires. Celle-ci résiste à Kaneie, ne craint pas de se chamailler avec lui, lui tient tête, lui refuse à l'occasion sa porte. En maint épisode, on voit qu'il existait entre eux une relation d'égal à égal, soit qu'ils s'affrontent, soit qu'on les sente complices, relations comme on n'en voit pas entre femmes et hommes dans le Genji.

On constate que la mère de Michitsuna entendait mener une vie personnelle, qu'elle ne craignait pas, par exemple, de voyager seule; indépendamment de sa liaison avec Kaneie, elle entretenait plusieurs relations avec le monde extérieur, avec d'autres femmes... et d'autres hommes. $Y$ aurait-il un lien de cause à effet avec cette relative autonomie qu'elle se donne vis-à-vis d'autrui ? On a en tout cas remarqué qu'il y avait beaucoup moins de keigo dans les Mémoires d'une Éphémère que dans le Genji².

Disons pour faire vite que, si Murasaki Shikibu est peut-être féministe en théorie, la mère de Michitsuna est féministe en pratique, mais seulement pour son propre compte: elle ne montre en effet aucune solidarité avec les femmes au nom d'une commune condition. Au contraire, elle oppose parfois son sort à celui des autres, plus heureuses selon elle, et elle explique ses malheurs par le caractère "extravagant " (mezurashi) de Kaneie.

10 Mais il faut revenir à notre propos: où peut se situer une influence des Mémoires d'une Éphémère sur le Genji monogatari, ou, plus exactement, un apport du Kagerō au Genji ? Et, question préalable, Murasaki Shikibu a-t-elle lu les Mémoires de la mère de Michitsuna?

11 On n'en tient aucune preuve formelle. Mais on considère la chose comme certaine : si "tout le monde admet que le Genji monogatari a reçu l'influence du Kagerō no nikki » , c'est qu'il est admis que Murasaki Shikibu l'a lu.

Divers arguments militent dans ce sens. Tout d'abord, Murasaki Shikibu était une parente éloignée de la mère de Michitsuna: son grand-père maternel, Fujiwara no Tamenobu, avait pour frère Tamemasa; or celui-ci avait épousé une sœur de l'auteur 
du Kagerō, et cette dernière entretenait d'excellentes relations avec lui : sa sœur vivait avec elle du temps que Tamemasa la fréquentait, et l'on sait que par la suite, même à une époque postérieure à celle que couvrent les Mémoires, ils restèrent en relation ${ }^{4}$.

D'autre part, Murasaki Shikibu fut, on le sait, au service de Shōshi, impératrice de l'empereur Ichijō一条天皇. Or Shōshi était une petite-fille de Kaneie, l'époux de l'auteur des Mémoires.

Enfin et surtout, la mère de Michitsuna jouissait d'une certaine notoriété en tant que poétesse ${ }^{5}$ et elle ne pouvait, à cet égard, échapper à l'attention de Murasaki Shikibu. Quatre de ses poèmes furent recueillis dans le Shūi shō 拾遺抄 (première sélection de Kintō 公任, préalable à la compilation du Shūi shū 拾遺集). Or, dans son Journal, Murasaki Shikibu dit que le Shūi shō fait partie d'un ensemble d'anthologies offert par Michinaga à sa fille Shōshi. Murasaki Shikibu l'a donc certainement lu ; ce faisant, elle a pu avoir connaissance, d'après le kotobagaki 詞書 (notice introductive) de l'un d'eux, des difficiles relations de la mère de Michitsuna avec Kaneie. Comment n'aurait-elle pas eu la curiosité d'aller voir de plus près, aux sources?

Que ce soit par l'une de ces voies ou par une autre, il est hautement vraisemblable que l'auteur du Genji, intéressée à tant d'égards par la mère de Michitsuna, se soit procuré les Mémoires d'une Éphémère, même si la diffusion de l'ouvrage n'était pas encore très large.

Sur quels points Murasaki Shikibu a-t-elle pu tirer profit de cette lecture ? On crédite notamment les Mémoires d'une Éphémère de deux apports considérables à la littérature en wabun 和文: tout d'abord, un procédé stylistique, à savoir la fusion des citations de poèmes dans la prose - fusion telle que ces citations peuvent demeurer inaperçues $d u$ lecteur non averti ${ }^{6}$. Le fait de ne pas annoncer les citations serait une nouveauté. Or Murasaki Shikibu recourra à ce procédé.

Avant de proposer un exemple, mentionnons le deuxième apport du Kagerō, sans doute beaucoup plus important: ce qu'on appelle parfois l'« analyse psychologique»; je dirais, plus concrètement, la fréquence, dans la prose narrative, du monologue intérieur dans lequel soit on s'efforce de décrypter les motivations réelles des actions ou des paroles des partenaires, soit on surprend dans ses propres réactions aux événements des contradictions, contradictions que l'on explicite, si l'on ne cherche pas toujours à les analyser. Voyons un premier exemple, où ces procédés apparaissent. L'auteur des Mémoires relate une visite de Kaneie, avec qui ses relations sont alors considérablement distendues ${ }^{7}$; il a passé la nuit chez elle.

Le matin venu : «En raison d'une obligation [je dois vous quitter, dit-il]... Mais dès demain ou après-demain... " Je n'en crus pas un mot, mais sans doute pensait-il ainsi m'apaiser. Ou bien cette visite était-elle la dernière? Je demeurais dans l'attente, et l'un après l'autre de nombreux jours passèrent à nouveau. C'est bien cela..., me disais-je, plus que jamais en proie à la tristesse (arishi yori mo keni mono zo kanashiki).

Les derniers mots sont empruntés textuellement à un poème de l'Ise monogatari ${ }^{8}$ :

\begin{tabular}{|l|l|l|}
\hline 忘るらむと & Wasuru-ran to & Ne m'oubliera-t-elle pas? \\
\hline 思ふ心の & Omohu kokoro no & Pense mon cœur \\
\hline うたがひに & Utagahi ni & En proie au doute. \\
\hline
\end{tabular}




\begin{tabular}{|l|l|l|}
\hline ありしよりけに & Arishi yori ke ni & Plus que jadis \\
\hline ものぞ悲しき & Mono zo kanashiki & J'en suis attristé \\
\hline
\end{tabular}

On relève ici le procédé d'insertion dans la prose de quelques mots empruntés à un waka, procédé qui permet d'abord de relever l'expression, mais qui, aux yeux du lecteur averti, doit aussi l'enrichir en suggérant, comme une sorte d'harmonique, la partie non citée du poème : ici, les trois premiers vers, où sont exprimés des doutes sur la fidélité de la / du partenaire.

On tient en outre, dans ce passage, un exemple de l'autre procédé signalé plus haut, procédé déjà fréquent dans les Mémoires et qui le deviendra plus encore dans le Genji: l'analyse que fait un personnage des propos d'un autre, en leur donnant une interprétation contraire à leur signification superficielle; en l'occurrence, la mère de Michitsuna interprète la promesse de Kaneie en attribuant à ce dernier des arrièrepensées.

\section{Autre exemple ${ }^{9}$ :}

Présentement, m'arrive un billet [de Kaneie], où je lis : «À un long temps d'interdit a succédé la cérémonie d'installation à mon nouveau poste, après quoi il a fallu observer une stricte réserve... Mais aujourd'hui, je brûle de... ». Il s'exprime avec une grande délicatesse. Je réponds, non sans me dire que, malgré l'empressement qu'il me donne à croire, il n'en fera certainement rien, dès lors que désormais je suis vouée à l'oubli ! et je ne m'arrête pas à ses propos, insouciance parfaite qui me consterne moi-même.

On observe ici un travail d'analyse sur les autres et sur soi-même. La mère de Michitsuna analyse le discours de Kaneie en fonction de la situation ainsi que, sans doute, de l'expérience: sous la surface des mots "délicats ", elle lit, comme dans le passage cité précédemment, l'indifférence de son partenaire. Précisons que, cette foisci, elle se trompe : le jour même, Kaneie vient lui rendre visite. À ce décalage instauré entre apparence et réalité (supposée) s'en ajoute un autre: la mère de Michitsuna, lassée des inégalités d'humeur et de la mauvaise foi de Kaneie, en arrive à l'« insouciance ", mais elle se dédouble pour observer avec surprise cette insouciance, fort nouvelle chez elle, qui signale l'œuvre du temps sur sa propre personne. Et à cette insouciance se substitue alors (ou se combine) un sentiment totalement différent : la consternation. C'est à propos de passages comme celui-ci qu'on peut parler d'« introspection » dans les Mémoires d'une Éphémère ${ }^{10}$.

Décryptage des propos d'autrui et dédoublement du moi - le moi qui ressent et le moi qui se regarde ressentir -: ces deux procédés sont, dit-on, une nouveauté dans l'histoire de la prose narrative japonaise. Ils sont en tout cas absents de la seule œuvre de caractère autobiographique en wabun antérieure aux Mémoires d'une Ephémère: le Tosa nikki ${ }^{11}$. Ils semblent également absents des œuvres de fiction alors existantes, mais il est vrai que nous n'en avons conservé que deux (le Taketori monogatari et l'Utsuho monogatari, au moins pour sa première partie).

Cependant, ce procédé reste malgré tout, si on le compare au Genji monogatari, embryonnaire. Murasaki Shikibu le développera considérablement ${ }^{12}$, et, de plus, elle le rendra plus complexe. En effet, on n'aura plus affaire, comme dans l'œuvre autobiographique qu'est le Kagerō, à un unique regard - celui du narrateur sur autrui (principalement Kaneie) et sur soi-même - mais à des regards croisés, ceux que chacun des personnages porte sur les autres ; au discours linéaire de la protagoniste/narratrice 
succédera une polyphonie de voix, voire une cacophonie, quand les interprétations ou les malentendus des divers personnages s'enchevêtrent.

Si autobiographie et roman diffèrent fondamentalement sur bien des points, ils ont en commun d'être des récits, de proposer une relation d'événements concernant des personnages. Sur ce plan, les Mémoires d'une Éphémère ont pu fournir à l'auteur du Genji un stock d'épisodes, de situations. En fait, depuis l'époque d'Edo, et même antérieurement, quand les commentateurs se sont attachés à la recherche des "sources» du Genji (on sait qu'ils ont cru en trouver soit dans les événements contemporains, soit dans les romans antérieurs), ils en ont aussi détecté un certain nombre dans les Mémoires. Poser le problème en termes de «sources » est une attitude bien entendu trop naïve, mais il n'en est pas moins vrai que, si on lit le Genji en ayant en tête les Mémoires d'une Éphémère, on est frappé par le nombre d'échos que l'on y retrouve; ne l'ayant pour ma part relu que jusqu'au chapitre "Suma ", j'en ai trouvé une bonne douzaine. Il est sans doute possible d'avancer que bien des situations évoquées par la mère de Michitsuna ont pu aiguillonner l'imagination de Murasaki Shikibu.

Par exemple, dès le Kakai-shō 河海抄 de Yotsutsuji Yoshinari 四辻善成 (XIV ${ }^{e}$ siècle), est émise l'idée que l'épisode de l'exil du Genji à Suma aurait été inspiré à Murasaki Shikibu par les troubles de l'ère Anna 安和の変 (969). Je n'entrerai pas dans les intrigues politiques de l'époque, mais on sait qu'elles aboutirent à l'exil du ministre de gauche Minamoto no Takaakira 源高明, accusé de complot, destitué et limogé13. Cette affaire marqua une étape importante dans la mainmise des Fujiwara sur le pouvoir. Or les troubles d'Anna sont évoqués dans le Kagerō no nikki, sur un ton dramatique :

[...] un événement survint dans le monde : quel crime avaient-ils donc commis? plusieurs personnages allaient être bannis, nouvelle qui fit si grand bruit qu'elle brouilla nos plans. Vers le $25^{e}$ ou $26^{e}$ jour du mois, Son Altesse le ministre de gauche, seigneur du palais de l'ouest [Minamoto no Takaakira], prend le chemin de l'exil. Chacun veut assister à son départ, le monde est ébranlé, on court en foule vers ce palais de l'Ouest [...]. Cette nouvelle me cause une douleur si vive que je pense défaillir; même moi, qui ignore le fond de l'affaire - à plus forte raison ceux qui en sont instruits -, il n'est personne qui ne mouille sa manche de larmes ${ }^{14}$.

26 Si la mère de Michitsuna mentionne ces événements dans ses Mémoires, c'est, dira-telle, parce qu'elle éprouve de la compassion pour l'exilé; elle en était d'ailleurs parente, et elle entretenait des rapports d'amitié avec son épouse, Aimiya 愛宮, à qui elle adressera un long et touchant poème.

Deux problèmes se posent, qu'il faut distinguer : Takaakira est-il une «clef » du Genji, du moins pour cet épisode? Murasaki Shikibu s'est-elle inspirée du Kagerō no nikki? S'agissant des clefs possibles, Abe Akio en propose une série, où figure Takaakira, qu'il juge d'ailleurs n'être pas le candidat le mieux placé ${ }^{15}$. Que Murasaki Shikibu se soit inspirée du Kagerō no nikki, nous avons dit que certains commentateurs anciens l'affirmaient, et, aujourd'hui, les co-éditeurs (dont Abe Akio) du Genji monogatari dans la collection de référence "Nihon koten bungaku zenshū » le suggèrent en passant, puisqu'ils relèvent dans les deux œuvres une similitude d'expression : «le monde fut ébranlé [par ce drame] » (Kagerō : ame no shita yusurite ; Genji : yo yusurite) ${ }^{16}$.

Mais je prendrai ces problèmes d'une autre façon. On sait que la notion même de clef unique n'a guère de sens dans la création romanesque. En outre, Murasaki Shikibu semble la récuser elle-même : elle dit explicitement que ce qui est arrivé au Genji est arrivé à beaucoup d'autres : «Tomber en disgrâce, en Morokoshi aussi bien que dans 
notre Empire, est pour un homme aussi éminent, qui en toute chose se distingue des autres, un accident inévitable ${ }^{17}$. » Elle fait donc de l'exil comme la marque du destin des hommes hors du commun, et l'on songe ici au scénario, si bien représenté au Japon, des 《errances d'un personnage de noble condition» (kishu ryūri tan 貴種流離譚), depuis Susanoo en passant par Michizane ${ }^{18}$.

Si l'on écarte la notion de clef unique, ainsi disqualifiée, qu'a bien pu tirer Murasaki Shikibu, en l'occurrence, des Mémoires d'une Éphémère? Nous venons de voir qu'elle jetait sur l'épisode une lumière valorisante : l'exil frappe les « hommes éminents ». Or c'est justement sous ce jour que l'auteur des Mémoires présente Takaakira. Jamais n'est évoquée la possibilité qu'il ait réellement commis une faute, de même que, si Murasaki Shikibu ne cache pas, dans certains passages, les tsumi 罪 du Genji (sa liaison avec Fujitsubo notamment), elle peut aussi, comme dans les lignes citées à l'instant, les ignorer totalement. De plus, la réaction de la mère de Michitsuna est la douleur. Or n'est-ce pas l'attitude que Murasaki Shikibu attribue aux proches de son héros, et qu'elle requiert du lecteur? Si l'auteur du Genji a tiré quelque chose de cet épisode des Mémoires d'une Éphémère, c'est, selon moi, l'exemple de l'utilisation d'un événement politique - l'exil d'un homme devenu persona non grata à la Cour -, dans une œuvre littéraire en wabun, une œuvre de femme, visant le même public que le monogatari ${ }^{19}$, comme ressort dramatique suscitant la compassion. Cet épisode a pu, à cet égard, être pour la romancière un stimulant. Notons cependant que, à la différence de la mère de Michitsuna, Murasaki Shikibu n'a pas évacué la dimension politique de l'événement: elle met en lumière, très nettement, les rivalités des factions à la Cour. Sachant que son récit serait lu comme une fiction, elle a pu, paradoxalement, décrire plus crûment la réalité.

Je relèverai un autre écho des Mémoires d'une Éphémère, dans ce même chapitre "Suma ». Il s'agit du passage où est décrite, après le départ du Genji pour l'exil, la douleur de Murasaki no ue. La romancière le fait en évoquant une expérience universelle : après la séparation d'avec un être cher, la douleur se ravive lorsque l'on retrouve un objet qui lui a appartenu ${ }^{20}$.

La dame de la Deuxième Avenue [Murasaki no ue] ne se levait plus de sa couche et s'abîmait dans une langueur sans fin [...]. Les objets dont il avait coutume d'user, une cithare dont il avait joué, le parfum d'un vêtement qu'il avait abandonné, tout cela elle le considérait comme si ç'avait été de quelqu'un qui venait de quitter ce monde.

31 À la lecture de cette page, me sont venus à l'esprit plusieurs passages du Kagerō, où est évoquée une expérience similaire. Il s'agit d'abord de deux épisodes parallèles entre eux, encore que séparés par cinq années ${ }^{21}$ : alors que Kaneie semble la délaisser et ne s'est pas montré de plusieurs jours, la mère de Michitsuna retrouve inopinément deux objets dont il se servait quotidiennement: l'eau de riz avec laquelle il se gominait les cheveux, et le remède qu'il prenait le matin et qui est resté glissé sous leur couche. Dans les deux cas, cette découverte fait sentir à la femme délaissée le passage du temps: la mère de Michitsuna compose à leur sujet un poème où elle exprime sa tristesse.

On voit cependant que Murasaki Shikibu, dans le passage que nous venons de citer, n'a pas mis en scène des objets aussi triviaux : il est question d'une cithare, d'un vêtement. Aussi cette scène me rappelle-t-elle plutôt deux autres passages du Kagerō où ces objets apparaissent dans un contexte similaire : l'auteur a vu mourir une mère très aimée, 
dans un temple de montagne ; elle a regagné ensuite sa demeure, où sa mère vivait avec elle ${ }^{22}$ :

Or donc, j'entreprends, dans mon déscuvrement, d'arranger le désordre que j'ai mis lors de notre départ pour le temple de montagne; à chaque fois que mes yeux s'arrêtent sur un objet dont elle se servait journellement, sur quelque lettre qu'elle avait préparée, je pense défaillir. Le jour que, affaiblie par la maladie, elle avait reçu les interdits, un révérend moine qui se trouvait là lui avait passé à l'épaule une étole ; restée sur elle, cette étole était atteinte par la souillure de la mort, et voilà que, maintenant, je la retrouvais parmi ses effets! Dans le dessein de la rendre, je me levai avant l'heure et commençai de rédiger: "Cette étole qui vous appartient... ", mais les larmes aussitôt vinrent brouiller ma vue.

Le second épisode se situe un an plus tard:

Une fois passé le jour anniversaire de sa mort, je retombai dans mon désœuvrement ordinaire, quand, en époussetant ma cithare, sans aucune intention d'en jouer, je la fis résonner ; je me disais : mais voilà que le temps des interdits est achevé23! ah, vraiment, rien ne dure...

La situation est, dans cette dernière scène, un peu différente, puisqu'il ne s'agit pas de la cithare de la disparue, mais de l'instrument dont la narratrice joue elle-même. Cependant, ici encore, c'est un objet qui vient rappeler l'heureux temps désormais aboli, celui où l'être aujourd'hui absent était là.

Ces passages ont-ils inspiré Murasaki Shikibu? Je me plais à l'imaginer. La similitude des objets est frappante - à cela près que l'étole, marquée par le contexte de la maladie, "devient » dans le Genji un simple vêtement. On retrouve des expressions semblables (en italiques dans les traductions), même si les mots sont différents (Kagerō : akekure toritsukahishi mono no gu nadomo ; Genji : motenarashitamahishi on-chōdo domo). Sans aller jusqu'à soutenir que Murasaki Shikibu a délibérément emprunté, puis transposé ces épisodes, je noterai que, sous la plume de ces deux écrivaines géniales, on relève un trait similaire : ce qui éveille les réminiscences d'un passé heureux mais aboli, ce ne sont pas seulement des objets vus, contemplés avec nostalgie. Dans le Kagerō, c'est une note échappée à la cithare, dans le Genji, le parfum d'un vêtement; dans les deux cas, il s'agit d'une expérience sensorielle subite, et évanescente. Le caractère fortuit de la sensation qui fait ressurgir le passé accentue la violence de l'émotion. Ce sont d'infimes notations comme celles-là qui sont la marque des grands écrivains : nous ne sommes pas très loin de Proust.

Mais on objectera peut-être que, dans les Mémoires d'une Éphémère, il s'agissait d'une mère, et non d'un époux, et que l'absente n'était pas partie au loin, mais morte. Or, curieusement, Murasaki Shikibu paraît répondre elle-même à ces objections et les balayer : certes, le Genji n'est pas mort, mais, comme nous l'avons vu plus haut, « tout cela, elle [Murasaki no ue] le considérait comme si ç'avait été de quelqu'un qui venait de quitter ce monde »; et, à la fin de ce passage, l'auteur insistera sur le fait que sa séparation d'avec le Genji est pire que la mort. Quant au fait que l'auteur du Kagerō n'a pas perdu un époux, mais sa mère, ne lit-on pas dans le Genji, quelques lignes après celles que nous avons citées, qu' « il lui avait tenu lieu de père et de mère » (chichihaha ni mo narite)?

Mon analyse ne convaincra peut-être pas; que les sceptiques y voient en tout cas un exemple instructif des dérives du commentaire, lorsqu'il fait flèche de tout bois au bénéfice d'une thèse.

Regardons un dernier exemple, qui permettra de mesurer les différences dans le traitement d'une situation analogue, lorsque l'on passe du Kagerō au Genji, différences 
révélatrices de la spécificité des deux œuvres. Il s'agit, dans le roman, de l'épisode fort connu dit de la « rivalité entre les chars » ou kuruma arasoi : lors de la fête de Kamo (fête dite des Mauves), ou, plus exactement, lors de la fête préalable de la purification de la Grande Prêtresse, les voitures d'Aoi et de Rokujō, venues admirer le cortège, se trouvent prises dans la cohue, et les gens d'Aoi bousculent la voiture de Rokujō, humiliant profondément celle-ci ${ }^{24}$. On a remarqué qu'une scène semblable figurait déjà dans l'Ochikubo monogatari25: dans ce roman, déjà, une querelle à propos de l'emplacement de voitures lors de la fête de Kamo dégénérait en un échange d'insultes, puis en une rixe opposant les gens des protagonistes, pour la plus grande humiliation de l'une des femmes en présence. Cependant, si Murasaki Shikibu a pu s'inspirer de l'Ochikubo monogatari dans la narration de l'épisode, les relations entre les personnages diffèrent considérablement : dans l'Ochikubo, il ne s'agit pas de deux rivales, mais de la jeune héroïne et de sa marâtre; d'autre part, le récit n'est pas, dans sa plus grande partie, focalisé sur les femmes, mais sur les hommes (d'un côté, l'amant de l'héroïne, de l'autre, le vieil oncle que la marâtre veut donner à celle-ci pour époux), et c'est entre eux qu'a lieu l'affrontement. En revanche, antérieurement à ce roman - sans doute rédigé dans les années 985-990 -, le Kagerō contenait une scène à certains égards plus proche. Citons ce passage ${ }^{26}$.

Environ cette époque-là - on était au $4^{e}$ mois - j'étais sortie pour assister à la Fête [des Mauves] et, chez l'autre personne [Tokihime] aussi, on était sortie. Pensant la reconnaître, j'arrêtai ma voiture en face de la sienne. Pour tromper l'ennui de l'attente, comme j'avais là quelques mandarines, j'y joignis des mauves et lui fis porter le tout, avec ces mots:

J'avais ouï dire

Que c'était jour de rencontre / mauves

Mais vous vous tenez là, indifférente... / mandarine ${ }^{27}$

Après un temps assez long vint cette réponse :

Ce sont vos rigueurs, madame,/ mandarine jaune, cannelier

Qu'aujourd'hui je vois ${ }^{28}$.

"Alors que depuis tant d'années, elle vous tient pour personne haïssable, fit observer quelqu'un de la compagnie, pourquoi préciser : "aujourd'hui" ? De retour au logis, je contai ce qui s'était passé ; alors, lui [Kaneie] : «Tiens ! elle n'a pas répliqué: "C'est de [les / vous] dévorer Que je sens l'envie" ? Et cela le mit en joie.

La situation, dans le Genji monogatari et dans les Mémoires, est presque identique: l'épouse en titre (Tokihime dans un cas, Aoi dans l'autre) et une rivale (la mère de Michitsuna dans un cas, la Dame de la Chambre Rokujō dans l'autre ${ }^{29}$ ) se trouvent en présence, chacune dans sa voiture, pour voir passer le cortège de la Fête. À lieu un affrontement, sans réconciliation finale. Par ailleurs, dans les deux cas, tout est relaté du point de vue de la femme qui n'a pas la préséance (la mère de Michitsuna, Rokujō).

Comment Murasaki Shikibu traite-t-elle l'épisode? Tout d'abord, elle le déploie superbement, en lui consacrant plusieurs pages. La mise en scène est développée, avec une multiplication des personnages : on a une description détaillée des voitures, de la querelle des valets, du cortège et des spectateurs. L'auteur de l'Ochikubo avait d'ailleurs déjà montré la voie en ce sens.

Mais il n'y a pas seulement développement. Si le propos est, dans les deux cas, de montrer un affrontement entre deux femmes, les rôles sont infléchis. Dans le Kagerō, les rivales se faisaient face, ou plutôt l'une d'elles, la mère de Michitsuna, faisait délibérément arrêter sa voiture vis-à-vis de celle de Tokihime, alors que, dans le Genji, c'est le hasard qui occasionne la rencontre; de même, la mère de Michitsuna provoque sa rivale, l'interpelle, en quelque sorte, et celle-ci répond sur le même ton, peu amène. 
Cependant, malgré l'agressivité latente, l'échange comporte un aspect ludique, qui désamorce la violence (comme le fera remarquer Kaneie). Au contraire, dans le Genji (comme dans l'Ochikubo), il n'y a ni affrontement direct ni dialogue entre les femmes. Dans l'Ochikubo, chacune s'épanchera par la suite; dans le Genji, elles resteront murées dans leur silence, enfermées dans leurs pensées. Certes, dans le Genji comme dans le Kagerō, un waka est composé, mais, au lieu d'un spirituel échange entre les rivales, il s'agit d'un poème soliloqué, seulement «murmuré » par Rokujō humiliée. Toute la violence du discours est reportée sur les valets: en s'insultant, ceux-ci semblent manipuler les deux femmes, qui, en tout cas, sont peintes comme totalement impuissantes.

41 Il faut dire un mot de la réaction de l'homme. Dans le Kagerō, la mère de Michitsuna raconte elle-même la scène à Kaneie, qui prend son parti (peut-être, tout simplement, parce que c'est elle qui est là) ; il plaisante et se moque de l'absente. La connivence se traduit par le fait que Kaneie se fait, après coup, acteur de l'épisode : il propose pour le tan-renga 短連歌 un autre complément. Dans le Genji, ce n'est pas par l'une des protagonistes que le partenaire masculin apprend ce qui s'est passé (toujours ce mutisme des femmes!), mais par des "gens » anonymes. Le Genji les plaint l'une et l'autre, fait même une tentative auprès de Rokujō, qui élude et se retranche dans le silence. Le «mot de la fin» du Genji, loin d'être un mot d'esprit joyeusement lancé comme celui de Kaneie, est un murmure bougon (uchi-tsubuyaki) : «Ah, pourquoi donc ne peuvent-elles vivre sans se heurter de la sorte?»; et le lendemain, lâchement, il sort... avec une troisième (Murasaki no ue) ${ }^{30}$.

Je dirai, par parenthèse, que, si le Genji ressemble pour certains traits à Kaneie (élégance, talent poétique, goût des femmes, alternance d'attentions délicates et de mufleries), il est beaucoup plus retors, plus pervers, et montre à l'occasion une grande violence à l'égard des femmes : rares sont sans doute les romans où les scènes de viol sont si nombreuses; il est aussi beaucoup moins drôle. Pour moi, si Murasaki Shikibu ne cesse de répéter qu'il est admirable, c'est pour compenser l'effet désastreux que sa conduite produit sur le lecteur..., et, à l'inverse, si l'auteur des Mémoires ne débusquait pas impitoyablement les travers de Kaneie, nous en garderions l'image d'un homme extrêmement séduisant. Dans les deux œuvres, narration et discours ne marchent pas de concert.

Pour en revenir à la scène de l'affrontement des deux femmes en char, on note encore que, si, dans les deux cas, le récit est conduit du point de vue de celle des femmes qui est située, dans sa relation matrimoniale, en position inférieure ${ }^{31}$, celle-ci, dans le Kagerō, se fait agresseur, prend une sorte de revanche et, allègrement, triomphe (elle s'acquiert les bonnes grâces de Kaneie). Dans le Genji, au contraire, la défaite de la femme humiliée, Rokujō, s'ajoute aux frustrations passées, et elle est encore avivée, lors du passage du cortège, par la froideur du Genji dont l'escorte ne la salue pas.

Dans cet épisode, et plus globalement, dans l'ensemble des deux œuvres (mais je généralise peut-être trop rapidement), l'atmosphère est radicalement différente : dans le Kagerō, on respire une sorte de fraîcheur. Ainsi, ici, les femmes jouent cartes sur table, elles se parlent (même pour échanger des propos assez vifs), elles rivalisent d'esprit. Le dernier mot de l'épisode n'est-il pas "mettre en joie »? Dans le Genji, au contraire, la scène se déroule dans un climat de violence extérieure, verbale et physique (insultes des valets, bousculade qui endommage la voiture de Rokujō), mais, paradoxalement, dans le silence des deux femmes : l'aspect feutré de leur confrontation 
la rend d'autant plus sombre. Le monde du Genji (je me surprends à utiliser le mot !) est noir.

Ajoutons que, outre la transformation apportée à l'épisode lui-même, Murasaki Shikibu en accentue l'importance par la place qu'elle lui donne dans l'ensemble du roman. Dans le Kagerō, il ne s'agissait que d'une scène isolée, sans incidence sur les relations entre les deux femmes - relations qui restent au long des années d'une froideur polie - alors que Murasaki Shikibu intègre l'épisode à une construction complexe, en fait le ressort d'un drame. On sait que les répercussions seront terribles : Rokujō, ulcérée, agira sous la forme d'un « esprit » ikiryō 生き霊 dévastateur, qui anéantira violemment sa rivale. Le traitement de cet épisode dans le Genji monogatari révèle donc un art de la mise en scène que ne possède pas à ce degré la mère de Michitsuna, mais aussi une propension à la "dramatisation », dans les deux sens de ce terme: les événements prennent un aspect dramatique, et chaque épisode vient, par ses répercussions, servir sa dramaturgie. Mais on touche là la différence entre autobiographie et roman: la présence d'un événement dans le récit peut, dans le premier cas, se justifier par le simple fait qu'il a eu lieu; dans le second, il doit s'intégrer à une trame, ou plutôt faire progresser l'histoire.

\section{NOTES}

1. Genji monogatari no sekai, Tōkyō, Tōkyō daigaku shuppan kai, 1964, p. 40.

2. Imuta Tsunehisa, « Kagerō no nikki no goi », Issatsu no kōza Kagerō no nikki, Tōkyō, Yūsei-dō, 1981, p. 325.

3. Ikeda Setsuko, « Kagerō no nikki shiron - Genji monogatari to no ruijiten to sōiten », Kotoba ga hiraku kodai bungaku shi, Suzuki Hideo (éd.), Tōkyō, Kasama shoin, 1999, p. 306-320.

4. Voir les kotoba-gaki de trois des poèmes du recueil personnel de la mère de Michitsuna, dont un figure aussi dans le Shūi shū (n 1339).

5. Sei Shōnagon cite dans le Makura no sōshi un poème de la mère de Michitsuna (Makura no sōshi, coll. « Nihon koten bungaku taikei », infra KBTK, Iwanami shoten, p. 317 ; trad. Beaujard, Notes de Chevet, Paris, Gallimard, 1966, p. 265).

6. Cf. Uemura Etsuko, « Genji monogatari to Kagerō no nikki », in Genji monogatari to joryū nikki, ouvrage collectif, Tōkyō, Musashino shoin, 1976. Elle relève dans le Kagerō soixante-six citations

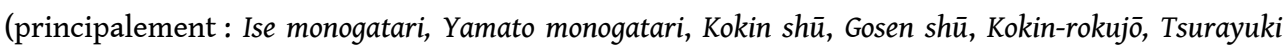
shū et autres recueils personnels).

7. Livre II, Tenroku 1 (970), $6^{\mathrm{e}}$ mois (coll. « Nihon koten bungaku zenshū », infra KBZ, Shōgakukan, p. 234). Nous citons ici notre traduction Mémoires d'une Éphémère, Bibliothèque de l'Institut des Hautes Études Japonaises, Collège de France, 2006, p. 94-95.

8. Chapitre XXI, traduction de Gaston Renondeau, Contes d'Ise, Gallimard, coll. "Connaissance de l'Orient ", p. 46.

9. Livre III, Tenroku 3 (972), $2^{\mathrm{e}}$ mois (KBZ, p. 307 ; traduction, p. 138).

10. Cf. Takada Hirohiko, Genji monogatari no bungaku shi, Tōkyō, Tōkyō daigaku shuppan kai, 2003, p. 13-14. 
11. Voir la traduction de R. Sieffert, Le Journal de Tosa, POF, 1993. On peut aussi ranger parmi les œuvres peut-être autobiographiques (ou partiellement autobiographiques) qui annoncent le Kagerō no nikki certains recueils poétiques personnels comme l'Ise shū 伊勢集 : on relève dans les kotobagaki de celui-ci, relativement développés, des embryons de discours intérieurs, mais ils n'ont pas la complexité de ceux du Kagerō.

12. Renvoyons à deux exemples entre cent: les interprétations que la "demoiselle d'Akashi » donne aux discours du Genji et ses délibérations sur la conduite à tenir à son égard dans le chapitre «Akashi» (KBZ, II, 243 sq.; trad. R. Sieffert, POF, I, 294), ou l'introspection (pleine de mauvaise foi) du Genji au début du chapitre «E awase» (KBZ, II, p. 360-362 ; trad. R. Sieffert, I, p. 349-351).

13. Sur cette affaire, voir le Dictionnaire historique du Japon, Tōkyō, Maison franco-japonaise/ Kinokuniya, à « Anna no hen ». L'attitude de Kaneie en cette occasion reste discutée.

14. Livre II, Anna 2 (969), $3^{\mathrm{e}}$ mois (KBZ, p. 206-207 ; traduction, p. 73-74).

15. Genji monogatari kenkyū josetsu, Tōkyō, Tōkyō daigaku shuppan kai, 1959, p. 630-634. Pour une présentation un peu plus détaillée de cette question, voir notre Michiyuki-bun - Poétique de l'itinéraire dans la littérature du Japon ancien, Paris, Maisonneuve et Larose, 1982, p. 295-296.

16. Chapitre « Suma», KBZ, vol. II, p. 176, note 8.

17. Chapitre « Suma », KBZ, vol. II, p. 202 ; trad. R. Sieffert, vol. I, p. 274.

18. Voir notre Michiyuki-bun, op. cit., p. 281-312.

19. Sur ce point, voir Kannotō Akio, "Kagerō no nikki to monogatari bungaku », Issatsu no kōza Kagerō no nikki, op. cit., p. 250.

20. KBZ, II, p. 181-182 ; trad. R. Sieffert, I, p. 261-262.

21. Livre I, Kōhō 3 (966), $8^{\mathrm{e}}$ mois (KBZ, p. 183-184; traduction, p. 55) et Livre II, Tenroku 2 (971), $6^{\mathrm{e}}$ mois (KBZ p. 259 ; traduction, p. 109).

22. Livre I, Kōhō 1 (964), $8^{\text {e }}$ mois (KBZ, p. 170 ; traduction, p. 46) et Kōhō 2 (965), $7^{\mathrm{e}}$ mois (p. 172-173; traduction, p. 48).

23. Il était considéré comme néfaste, pendant le deuil, de jouer de la cithare.

24. Chapitre « Aoi » (KBZ, II, p. 14-20 ; trad. R. Sieffert, I, p. 178-182).

25. Je remercie Daniel Struve de me l'avoir rappelé.

26. Kōhō, 3 (966). KBZ, p. 180-181; traduction, p. 53. Selon Joshua Mostow, il y aurait reprise délibérée, dans les trois œuvres, de la même situation, peut-être plus anciennement présente dans la littérature japonaise ("The Amourous Statesman and the Poetess: The Politics of Autobiography and the Kagerō Nikki », Japan Forum (Oxford), 4-2, octobre 1992, p. 305-315. Je remercie Michel Vieillard-Baron de m'avoir signalé et procuré cet article.)

27. Ahu hi to ka / kikedomo yoso ni / tachibana no. Dans la traduction, la barre oblique sépare les deux traductions possibles des mêmes syllabes.

28. Kimi ga tsurasa wo / Kehu koso ha mire. Pour l'analyse de ce tan-renga, voir Terada Sumie, Figures poétiques japonaises - La genèse de la poésie en chaîne, Bibliothèque de l'Institut des Hautes Études Japonaises, Collège de France, 2004, p. 161-162.

29. La mère de Michitsuna est aussi l'épouse de Kaneie; mais elle est venue plus tard que Tokihime, et Kaneie montre toujours plus d'égards pour cette dernière. Rokujō n'est qu'une maîtresse du Genji.

30. Dans l'Ochikubo, chacune des deux femmes s'épanche au retour, la marâtre pour se plaindre auprès de son époux, l'héroïne, compatissante, pour reprocher à son époux sa violence. Sur ce point, les trois textes diffèrent totalement.

31. Dans l'Ochikubo, le point de vue change constamment ; par ailleurs, rien n'est dit, durant toute la scène, de l'héroïne. 


\section{RÉSUMÉS}

De nombreuses similitudes existent entre le Genji monogatari et un récit de fiction légèrement antérieur, le Kagerō no nikki ( $\mathrm{x}^{\mathrm{e}}$ siècle). Celles-ci sont-elles fortuites, ou Murasaki Shikibu en avaitelle connaissance alors qu'elle écrivait?

Many similarities exist between the Genji monogatari and the Kagerō no nikki, an almost contemporary novel (10th century). Is this coincidental or was Murasaki Shikibu aware of the Kagerō no nikki when she wrote her novel?

\section{INDEX}

Thèmes : littérature

キーワード : jiden 自伝, tenjō -- tenjōbito 殿上・殿上人, josei -- mibun 女性・身分, Genji monogatari 源氏物語, Kagerō no nikki 蜻蛉日記, monogatari 物語, Murasaki Shikibu 紫式部 (v. 973-v. 1014 ou 1025), Heian jidai 平安時代 （794-1185), bungaku 文学

Keywords : Autobiography, Courts and Courtiers, Genji monogatari, Heian, Kagerō nikki, Literature, monogatari, Murasaki Shikibu (v. 973-v. 1014 or 1025), Women -- Social Conditions, Tale of Genji

Mots-clés : autobiographie, cour et courtisans, femmes -- conditions sociales, Genji monogatari, Kagerō nikki, Mémoire d'une éphémère, monogatari, Murasaki Shikibu (v. 973-v. 1014 ou 1025), Dit du Genji, écriture

Index chronologique : Heian 